\title{
Gluconic acid aqueous solution: A bio-compatible media for one- pot multicomponent synthesis of dihydropyrano [2,3-c] pyrazoles
}

\author{
Furqan Diwan Mubarak H. Shaikh $\odot$, Samreen Fatema $\odot$ and \\ Mazahar Farooqui
}

Department of Chemistry, Dr. Rafiq Zakaria, college for women, Aurangabad-431001, India.

(Received August 24, 2019; Revised December 16, 2019; Accepted December 19, 2019)

\begin{abstract}
A new facile, green and efficient protocol was developed for synthesis of Dihydropyrano [2,3-c] pyrazoles using gluconic acid aqueous solution (GAAS), as catalyst and solvent under environmentally friendly conditions. The advantageous features of this methodology are the environmentally benign character, operational simplicity, high yield processing (91-96\%), easy handling; reaction medium can be recycled and reused several times without significant loss of its efficiency. The synthesized compounds were evaluated for antimicrobial and antioxidant activity and also analyzed for ADME properties. The structure of compounds has been confirmed by IR, 1H NMR, 13C NMR, Mass spectrometry and elemental analysis.
\end{abstract}

Keywords: Dihydropyrano [2,3-c] pyrazoles; gluconic acid aqueous solution (GAAS); green media; antimicrobial; antioxidant; ADME. @ 2019 ACG Publications. All rights reserved.

\section{Introduction}

Gluconic acid is an organic compound with molecular formula $\mathrm{C}_{6} \mathrm{H}_{12} \mathrm{O}_{7}$ and it is abundantly available in plants, fruits, and other foodstuffs, such as rice, meat, dairy products, wine, honey, and vinegar. In particular, the nonhazardous nature of gluconic acid also allows its use in the formulation of food, pharmaceutical and hygienic products. ${ }^{1}$ Gluconic acid aqueous solution (GAAS) is a weak acidic aqueous solution. It is noncorrosive, nonvolatile, stable, inexpensive industrial product and largely available in the market. GAAS is a effective promoting medium for organic reactions, such as the Michael addition of indoles to $\alpha, \beta$-unsaturated ketones, the electrophilic ring-opening reaction of 3,4-dihydropyran with indoles and Friedel-Crafts alkylation of electron-rich aromatics with benzyl alcohols. ${ }^{2}$ Whereas in our present work we have demonstrated, the use of GAAS as a green reaction media for the bicyclic ring closure reaction using one pot four component system.

\footnotetext{
* Corresponding author: E-mail: mazahar64@@rediffmail.com ; Phone: +919422214785
}

The article was published by ACG Publications 
In medicinal chemistry and drug designing, Dihydropyrano [2,3-c]pyrazoles is became the first choice of researchers and scientist due to its potential biological activity. Therefore it becomes an interesting template for medicinal chemistry research. Most of these compounds, are well known for antioxidant, ${ }^{3}$ antimicrobial, ${ }^{4}$ insecticidal, ${ }^{5}$ molluscicidal, ${ }^{6}$ analgesic, ${ }^{7}$ anti-inflammatory agents ${ }^{8}$ and some of their analogues act as vasodilators, hypotensive, ${ }^{9}$ hypoglycemic and anticancer agents. ${ }^{10}$ They are also potential inhibitors of human Checkpoint kinase-1 (Chk1) ${ }^{11}$ (Figure 1). Furthermore, they play a significant role as crucial synthetic intermediates. ${ }^{12}$<smiles>Cc1n[nH]c2c1C(c1ccco1)C(C#N)=C(N)O2</smiles>

Molluscicide

6-amino-4-(furan-2-yl)-1,4-dihydro-3methylpyrano[2,3-c]pyrazole-5-carbonitrile<smiles>Cc1n[nH]c2c1C(c1ccc(O)c(O)c1)C(C#N)=C(N)O2</smiles>

Inhibitors of human Chk1 kinase

6-amino-1,4-dihydro-4-(3,4-dihydroxyphenyl) 3-methylpyrano[2,3-c]pyrazole-5-carbonitrile

Figure 1. Biologically active dihydropyrano [2,3-c]-pyrazoles

Thus considering the different potential therapeutic activity of pyrano [2,3-c]pyrazoles heterocyclic compounds, various methodologies for synthesis of Dihydropyrano [2,3-c]pyrazoles have been reported in the literature. These methodologies have shown many good results in many instances. However, some of the synthetic strategies have limitation in term of using metal catalyst, expensive reagents, long reaction time, environmental hazard, harsh reaction conditions, tedious workup procedure, unsatisfactory yield and use of homogeneous catalyst. Which are difficult in separation from reaction mixture inspite of many reported methods for the synthesis Dihydropyrano [2,3c]pyrazoles derivatives. The development of new synthetic strategy using easily accessible catalyst and mild sustainable alternative reaction condition still demand a lot of researcher's attention.

Recently, One pot four-component reactions of aldehydes, 1,3-dicarbonyl compounds, malononitrile, and hydrazine have been developed for the synthesis of pyranopyrazoles using triphenylphosphine, ${ }^{13}$ urea, ${ }^{14}$ ionic liquid, ${ }^{15}$ water containing a catalytic amount of piperidine, ${ }^{16}$ CTACl,${ }^{17}$ heteropolyacids,${ }^{18}$ microwave,${ }^{19}$ piperazine,${ }^{20} \mathrm{~N}$-methylmorpholine, ${ }^{21}$ L-proline,${ }^{22}$ alumina,${ }^{23}$ per-6-amino- $\beta$-cyclodextrin, ${ }^{24}$ sodium benzoate, ${ }^{25}$ amberlyst $\mathrm{A} 21,{ }^{26}$ glycine, ${ }^{27}$ imidazole, ${ }^{28}$ and $\mathrm{I}_{2}{ }^{29}$ Although these methods are quite satisfactory, some of them suffer from the absence of green chemistry and have been associated with several shortcomings, such as the use of volatile and hazardous organic solvents, low yields, extended reaction time, high temperature and tedious procedure for the preparation of catalysts. Considering the significance of Dihydropyrano [2,3$c$ ]pyrazoles derivatives in pharmaceutical and medicinal fields, and in continuation of earlier work, ${ }^{30}$ the development of general, economically and environmentally benign synthetic methodologies for these heterocyclic is highly desirable.

\section{Experimental}

\subsection{Chemical Material and Apparatus}

All the reagents and solvents used for the synthesis were purchased from Sigma Aldrich, Spectrochem and Molychem and were used as such without further purification. The melting points of all compounds were determined on a Toshniwal apparatus and are uncorrected. IR spectra were recorded on a Shimadzu FTIR-8400S spectrophotometer using $\mathrm{KBr}$ pellets. ${ }^{1} \mathrm{H}$ and ${ }^{13} \mathrm{C}$ NMR spectra were recorded in DMSO- $\mathrm{d}_{6}$ using TMS as an internal standard on a Bruker spectrophotometer, 
respectively. Mass spectra of representative compounds were recorded on JEOL SX-102 spectrometer at $70 \mathrm{eV}$. Elemental microanalyses were carried out on a Carlo Erba1108 CHN analyzer. Thin layer chromatography was performed on pre-coated silica gel $60 \mathrm{~F}_{254}$ aluminium sheets (E. Merck, Germany) using various solvents systems and spots were identified by UV light and Iodine.

\subsection{Biological Activity}

\subsubsection{Antibacterial Activity}

Minimum inhibitory concentration (MIC) values for bacteria determined according to the twofold broth micro-dilution method using Muller-Hinton broth in 96-well micro-test plates recommended by National Committee for Clinical Laboratory Standards (NCCLS) guidelines. ${ }^{31 a, b}$ The antimicrobial susceptibility testing of newly synthesized compounds was performed In Vitro against bacterial strains viz., Gram-positive Staphylococcus Aureus (ATCC No. 29737), Micrococcus Luteus (ATCC No. 398) and Gram negative Escherichia Coli (NCIM No. 2256) and Pseudomonas Fluorescens (NCIM No. 2173), respectively, to find out minimum inhibitory concentration (MIC). The MIC was defined as the lowest concentrations of compound that completely inhibit the growth of each strain. Serial twofold dilutions of all samples were prepared in triplicate in micro titer plates and inoculated with suitably prepared cell suspension to achieve the required initial concentration. Serial dilutions were prepared for screening. Dimethylsulfoxide (DMSO) was used as solvent control. Ampicilin \& kanamycin were used as a standard antibacterial drug. The concentration range of tested compounds and standard was $128-0.5 \mu \mathrm{g} / \mathrm{mL}$. The plates were incubated at $37{ }^{\circ} \mathrm{C}$ for all microorganisms; absorbance at $595 \mathrm{~nm}$ was recorded to assess the inhibition of cell growth after $24 \mathrm{~h}$. The compounds which are showing promising antibacterial activity were selected for MIC studies. The MIC was determined by assaying at $128,64,32,16,8,4,2,1$ and $0.5 \mu \mathrm{g} / \mathrm{mL}$ concentrations along with standards at the same concentrations.

\subsubsection{Antifungal Activity}

The antifungal activity was evaluated against five human pathogenic fungal strains, such as Candida albicans (NCIM 3471), Fusarium oxysporum (NCIM 1332) and Aspergillus flavus (NCIM 539), which are often encountered clinically and were compared with standard drug fluconazole \& miconazole. Minimum inhibitory concentration (MIC) values were determined using standard agar method as per CLSI (formerly, NCCLS) guidelines (Approved Standard M7-A6, vol. 23. 2003). ${ }^{32 a, b}$ The standards used in the study were dissolved in a suitable solvent. The primary solutions were further diluted to the final strength using test medium. The medium yeast nitrogen base (Himedia, India) was dissolved in Phosphate buffer $\mathrm{pH} 7$ and it was autoclaved at $110{ }^{\circ} \mathrm{C}$ for 10 minutes. The suitable concentration of standards was incorporated in the medium. The fungal strains were freshly subcultered on to Sabouraud dextrose agar (SDA) and incubated at $25{ }^{\circ} \mathrm{C}$ for $72 \mathrm{~h}$. The fungal cells were suspended in sterile distilled water and diluted to get 105 cells $/ \mathrm{mL}$. $10 \mu \mathrm{L}$ of standardized suspension was inoculated onto the control plates and the media incorporated with the antifungal agents. The inoculated plates were incubated at $25{ }^{\circ} \mathrm{C}$ for $48 \mathrm{~h}$. The readings were taken at the end of 48 and $72 \mathrm{~h}$.

\subsubsection{Antioxidant Activity}

Antioxidant activities of the synthesized compounds 5a-q were measured using 2,2-diphenyl1-picrylhydrazyl (DPPH) radical scavenging assay. ${ }^{33}$ The hydrogen atom or electron donation ability of some compounds were measured from the bleaching of the purple colored methanol solution of DPPH. The spectrophotometric assay uses the stable radical DPPH as a reagent. $1 \mathrm{~mL}$ of various concentrations of the test compounds $(5,10,25,50$ and $100 \mu \mathrm{g} / \mathrm{mL})$ in methanol was added to $4 \mathrm{~mL}$ of $0.004 \%(\mathrm{w} / \mathrm{v})$ methanol solution of DPPH. The reaction mixture was incubated at $37{ }^{\circ} \mathrm{C}$. The scavenging activity on DPPH was determined by measuring the absorbance at $517 \mathrm{~nm}$ after $30 \mathrm{~min}$. All tests were performed in triplicate and the mean values were entered. The percent of inhibition (I $\%$ ) of free radical production from DPPH was calculated by the following equation $\%$ of scavenging $=$ 
[(Acontrol - Asample)/(Asample $\times 100)]$ Where, Acontrol is the absorbance of the control (DPPH radical without test sample) Asample is the absorbance of the test sample (DPPH radical with test sample). The control contains all reagents except the test samples. A lower IC50 value indicates the greater antioxidant activity. The IC50 (concentration required to scavenge 50\% of the radicals) were calculated to evaluate the potential antioxidant activities. Butylated hydroxytoluene (BHT) has been used as a standard drug for the comparison of antioxidant activity and the observed results are summarized in Table 3.

\subsubsection{Computational Study}

\subsubsection{ADME Properties}

The success of a drug is determined not only by good efficacy but also by an acceptable ADME (absorption, distribution, metabolism and excretion) profile. In the present study, we have calculated molecular volume (MV), molecular weight (MW), logarithm of partition coefficient (miLog $\mathrm{P}$ ), number of hydrogen bond acceptors (n-ON), number of hydrogen bonds donors (n-OHNH), topological polar surface area (TPSA), number of rotatable bonds (n-ROTB) and Lipinski's rule of five $^{34}$ using Molinspiration online property calculation toolkit. ${ }^{35}$ Absorption (\% ABS) was calculated by: $\%$ ABS $=109-(0.345 \times \text { TPSA })^{36}$ Drug-likeness model score (a collective property of physicchemical properties, pharmacokinetics and pharmacodynamics of a compound is represented by a numerical value) was computed by MolSoft ${ }^{37}$ software.

\section{Result and discussion}

\subsection{Chemistry}

A facile, economic, green and environmentally being protocol, was developed for one-pot MCR of aldehyde, malononitrile, hydrazine hydrate and ethyl acetoacetate (Scheme 1). Successful implementation of GAAS reaction medium for an efficient and rapid synthesis of pyrano [2,3c]pyrazole derivatives has been described. Higher product yields with shorter reaction time, reusable and economical catalytic system, and consistent performance on large scale make this synthetic strategy an attractive one (Scheme 1).

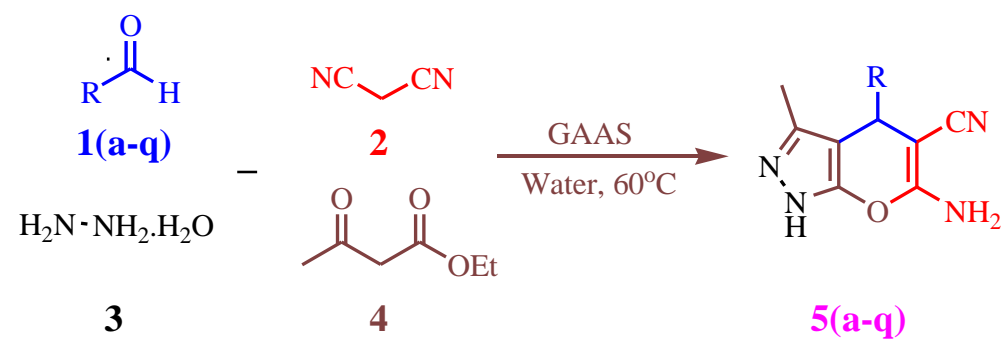

Scheme 1. Synthesis of pyrano[2,3-c]pyrazole derivatives.

In order to optimize the reaction conditions, initially we carried out the reaction between benzaldehyde (1) (1 mmol), malononitrile (2) $(1 \mathrm{mmol})$, hydrazine hydrate $(\mathbf{3 a})(1 \mathrm{mmol})$ and ethyl acetoacetate (4) $(1 \mathrm{mmol})$ as a model reaction. Before proceeding towards the actual experimental part, a thorough analysis of the mechanistic path leading to the formation of the desired pyrano [2, 3-c] pyrazole system was performed. This detailed study revealed that the first two steps involved in the reaction path i.e. formation of Knoevenagel condensation product $\mathbf{A}$ and pyrazolone $\mathbf{B}$ can be achieved either under solvent-free condition or using water as a reaction medium, that even in the absence of catalyst. The only challenge was to achieve the desired product $\mathbf{C}$ by cycloaddition of $\mathbf{A}$ and $\mathbf{B}$ (Figure 2). 

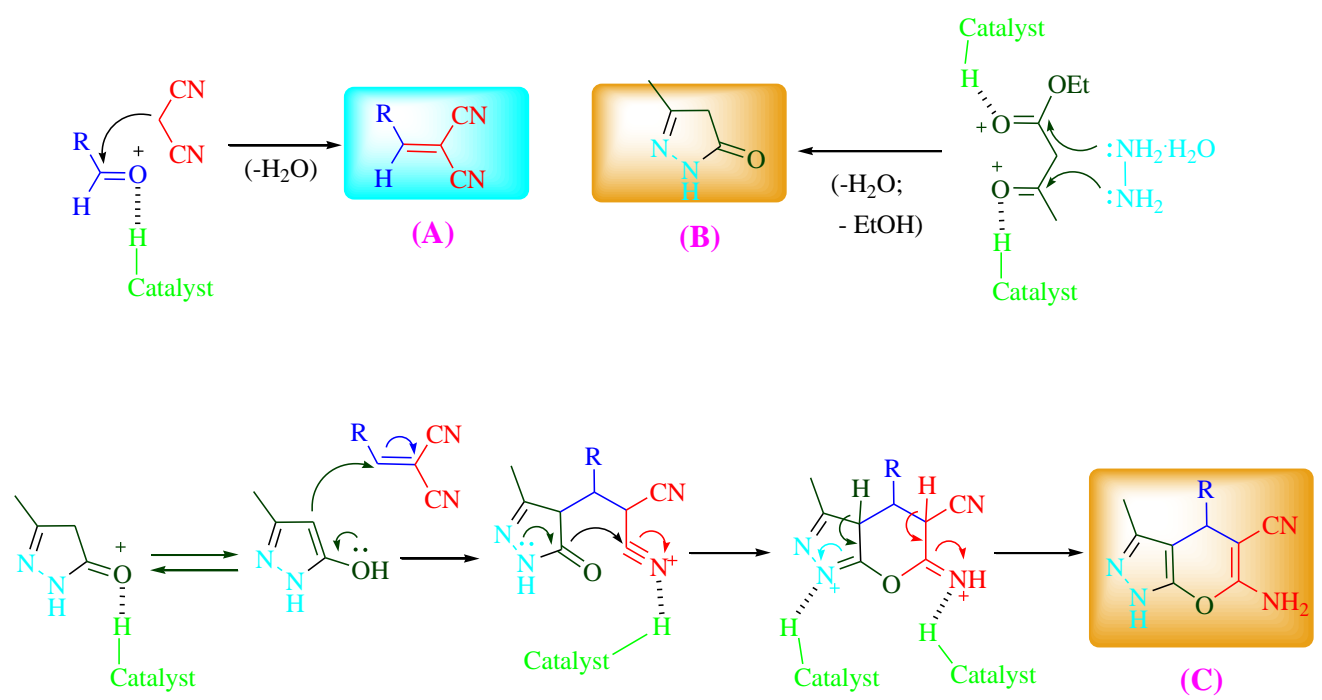

Figure 2. Proposed mechanism for GAAS catalyzed synthesis of pyrano [2,3-c] pyrazoles.

Considering the significance of green chemistry concept, model reaction was carried out initially under solvent-free and catalyst-free conditions at RT and higher temperature $\left(100{ }^{\circ} \mathrm{C}\right)$ for 60 min. But, formation of the desired product was not observed (Table 1, entries 1-2). During the study, model reaction was performed using water as a reaction medium at different temperatures. To our surprise, reaction in aqueous media at reflux conditions proceeds towards the desired product in $40 \%$ yield (Table 1, entry 4). Similarly, reaction carried out in different solvents like acetonitrile, THF, DMSO and methanol; trace amount of products were detected (Table 1, Entry 5-8).

Table 1. Screening of solvent and GAAS at different conditions ${ }^{\mathrm{a}}$

\begin{tabular}{ccccc}
\hline Entry & Solvent & Temp $\left({ }^{\circ} \mathbf{C}\right)$ & Time $(\mathbf{h})$ & Yield $^{\mathbf{b}}(\boldsymbol{\%})$ \\
\hline 1 & Neat & RT & 3 & Trace \\
2 & Neat & 100 & 3 & Trace \\
3 & Water & RT & 2 & Trace \\
4 & Water & Reflux & 2 & 40 \\
5 & $\mathrm{CH}_{3}$ CN & Reflux & 3 & Trace \\
6 & THF & Reflux & 3 & Trace \\
7 & DMSO & Reflux & 3 & Trace \\
8 & Methanol & Reflux & 3 & Trace \\
09 & Acetic acid & Reflux & 3 & 48 \\
10 & Citric acid-DMU (40:60) & Reflux & 2 & 54 \\
11 & L-(+)-Tartaric acid-DMU(30:70) & Reflux & 2 & 58 \\
12 & D-(-)-Fructose-DMU(70:30) & Reflux & 2 & 55 \\
13 & GAAS & RT & 2 & 30 \\
14 & GAAS & 40 & $60 \mathrm{~min}$ & 70 \\
15 & GAAS & 60 & $30 \mathrm{~min}$ & 95 \\
16 & GAAS & 80 & $30 \mathrm{~min}$ & 95 \\
\hline
\end{tabular}

${ }^{\mathrm{a}}$ Reaction conditions: 1a $(1 \mathrm{mmol}), 2(1 \mathrm{mmol}), 3(1 \mathrm{mmol}), 4(1 \mathrm{mmol})$ and solvent $(5 \mathrm{~mL})$

${ }^{\mathrm{b}}$ Isolated Yields.

Therefore, to improve the yield, it was thought that intervention of catalyst is necessary. Acetic acid was examined and displayed less efficiency (Table 1, Entry 9). When the reaction proceeded in low melting mixtures, such as citric acid-dimethylurea (DMU), L-(+)-tartaric acid-DMU, and D-(-)-Fructose-DMU slightly improved yields obtained (Table 1, Entry 10-12). With these results in hand, our next objective was to increase the product yield obtained in the earlier study. The only way in mind was the addition of a suitable catalyst which could enhance efficiency of the present method in terms of product yield as well as reaction time. It was decided to utilize GAAS system for our reaction. When the model reaction was performed in this reaction medium, at RT, 40, 60 and 
$80^{\circ} \mathrm{C}$; we were delighted to know that the reaction was completing within only $30 \mathrm{~min}$ at 60 and $80^{\circ} \mathrm{C}$ affording the product in good yield (95\%) (Table 1, Entry 15-16). Considering the effective catalytic activity, GAAS was preferred as a catalyst as well as solvent of choice for subsequent optimization studies.

Reason behind the success of GAAS bringing the reaction in its favor may be hydrophobic interactions which induce favorable aggregation of organic substrates in water. Due to this the organic substrates aggregate and result in their increased concentration which leads to fast collisions of the reactants thus leading to the formation of desired product in very short reaction times. Furthermore, exceptional performance of gluconic acid as an effective organic reaction medium in water could be attributed to the fact that the organic substrates get adsorbed to the gluconic acid by hydrophobic interactions between the surface of the gluconic acid and the organic molecule. In addition, the surface area available for a reaction (surface area of the gluconic acid) in such a system is quite large compared with that of the interface in a conventional liquid-liquid biphasic system.<smiles>Cc1n[nH]c2c1C(c1ccccc1)C(C#N)=C(N)O2</smiles><smiles>Cc1n[nH]c2c1C(c1ccc(F)cc1)C(C#N)=C(N)O2</smiles><smiles>Cc1n[nH]c2c1C(c1cccc([N+](=O)[O-])c1)C(C#N)=C(N)O2</smiles>

$5 \mathbf{k}$<smiles>Cc1ccc(C2C(C#N)=C(N)Oc3[nH]nc(C)c32)cc1</smiles>

5 b<smiles>Cc1n[nH]c2c1C(c1cccc(Br)c1)C(C#N)=C(N)O2</smiles><smiles>Cc1n[nH]c2c1C(c1ccc([N+](=O)[O-])cc1)C(C#N)=C(N)O2</smiles>

$5 \mathbf{l}$<smiles>COc1ccc(C2C(C#N)=C(N)Oc3[nH]nc(C)c32)cc1</smiles><smiles>Cc1n[nH]c2c1C(c1ccc(Br)cc1)C(C#N)=C(N)O2</smiles>
$5 \mathrm{~h}$<smiles>COc1ccc(C2C(C#N)=C(N)Oc3[nH]nc(C)c32)cc1OC</smiles>
$5 \mathrm{~m}$<smiles>Cc1n[nH]c2c1C(c1ccc(Cl)cc1)C(C#N)=C(N)O2</smiles><smiles>Cc1n[nH]c2c1C(c1ccccc1Cl)C(C#N)=C(N)O2</smiles><smiles>Cc1n[nH]c2c1C(c1ccc(O)cc1)C(C#N)=C(N)O2</smiles>
$5 i$<smiles>Cc1n[nH]c2c1C(c1ccccc1[N+](=O)[O-])C(C#N)=C(N)O2</smiles><smiles>[SiH3]</smiles><smiles>COc1cc(C2C(C#N)=C(N)Oc3[nH]nc(C)c32)ccc1O</smiles>

$5 n$<smiles>Cc1n[nH]c2c1C(c1ccc(N(C)C)cc1)C(C#N)=C(N)O2</smiles>

50<smiles>Cc1n[nH]c2c1C(c1ccco1)C(C#N)=C(N)O2</smiles>

$5 p$<smiles>Cc1n[nH]c2c1C(c1cccs1)C(C#N)=C(N)O2</smiles>

$5 q$

Figure 3. Structures of Dihydropyrano [2,3-c]pyrazole derivatives 5(a-q)

It should be noted that, because of the immiscibility of GAAS with non-polar organic solvent, the formed product could be easily extracted from the GAAS phase with an appropriate organic solvent. After completion of the reaction, the reaction mixture was cooled to room temperature and extracted with ethyl acetate. The recovered gluconic acid aqueous solution was then subjected to the next run in the model reaction and could be reused at least four times without significant loss of activity (Table 2). 
Table 2. Results for the recyclability of the GAAS

\begin{tabular}{cccc}
\hline Entry & Cycle & Isolated Yield\% & Recovered GAAS (w/w \%) \\
\hline 1 & 1 & 95 & 98 \\
2 & 2 & 93 & 96 \\
3 & 3 & 92 & 95 \\
4 & 4 & 90 & 93 \\
5 & 6 & 90 & 90 \\
\hline
\end{tabular}

To demonstrate the efficiency and the applicability of the developed method, reaction was performed with variety of electronically divergent aryl aldehydes under optimized reaction conditions and no obvious electronic effects of the substituent present on the aromatic ring of aldehyde was observed, affording the products in each case with excellent yields (Supporting Information). Structures of all the synthesized compounds shown in Figure 3.

The synthesized compound may exist in $1 H$ or $2 H$ forms $(\mathbf{5 a}$ or $\mathbf{5 b})$. Structure $\mathbf{5 a}$ is established through inspection of result by NOE experiments. Thus upon irradiation into the methyl signal a strong effect for the $\mathrm{NH}$ proton was revealed. This is a proof for the major position of the $\mathrm{NH}$ proton on $\mathrm{N}-2$. A fast equilibrium with $\mathbf{5 b}$ as minor components cannot be excluded at room temperature (Figure 4 ). ${ }^{38}$

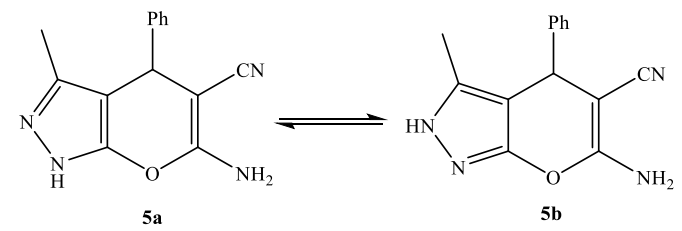

Figure 4. The synthesized compound may exist in $1 H$ or $2 H$ tautomeric forms (5a or $\mathbf{5 b})$

\subsection{General Procedure for the Synthesis Dihydropyrano [2,3-c]pyrazoles 5(a-q)}

A mixture of aromatic aldehyde $\mathbf{1}(\mathbf{a}-\mathbf{q})(1 \mathrm{mmol})$, malononitrile (2) $(1 \mathrm{mmol})$, hydrazine hydrate (3) $(1 \mathrm{mmol})$ and ethyl acetoacetate (4) $(1 \mathrm{mmol})$ in gluconic acid aqueous solution (GAAS) $(5 \mathrm{~mL})$ were taken in a $50 \mathrm{~mL}$ round-bottomed flask. The resulting mixture was stirred at $60^{\circ} \mathrm{C}$ for a period as indicated in Table 3. After completion of the reaction (monitored by TLC), the solid obtained was collected by simple filtration and washed successively with water. The crude product was purified by crystallization from ethanol. The products $\mathbf{5}(\mathbf{a}-\mathbf{q})$ were confirmed by comparing the physical and spectral data with those of the reported compounds.

6-Amino-3-methyl-4-phenyl-1,4-dihydropyrano[2,3-c]pyrazole-5-carbonitrile (5a): Time: $30 \mathrm{~min}$; yield: 95\%; Melting point: $241-243^{\circ} \mathrm{C} ;{ }^{15}$ FTIR $\left(\mathrm{KBr}, \mathrm{cm}^{-1}\right): v=1654(\mathrm{C}=\mathrm{C}), 2193(\mathrm{C} \equiv \mathrm{N}), 3398\left(\mathrm{NH}_{2}\right)$. ${ }^{1} \mathrm{H}$ NMR (400 MHz, DMSO-d 6 ) $\delta$ ppm: $2.06\left(\mathrm{~s}, 3 \mathrm{H}, \mathrm{CH}_{3}\right.$ ), 4.59 (s, 1H, CH), 5.48 (s, 1H, NH), 7.237.47 (m, 5H, Ar-H), 10.48-11.6 (bs, $\left.2 \mathrm{H}, \mathrm{NH}_{2}\right) ;{ }^{13} \mathrm{C}$ NMR (400 MHz, DMSO-d 6 ) $\delta p p m: 8.82,34.74$, 57.77, 94.70, 96.54, 119.71, 127.38, 134.69, 134.85, 153.85, 157.02, 159.50; Mass (LC-MS) $\mathrm{m} / \mathrm{z}$ : $251.2\left(\mathrm{M}^{-}\right)$; Elemental analysis calculated for $\mathrm{C}_{14} \mathrm{H}_{12} \mathrm{~N}_{4} \mathrm{O}$ : C $(66.65 \%), \mathrm{H}(4.79 \%), \mathrm{N}(22.21 \%)$. Found: C (66.57\%), H (4.63\%), N (22.13\%).

6-Amino-1,4-dihydro-3-methyl-4-p-tolylpyrano[2,3-c]pyrazole-5-carbonitrile (5b): Time: 30 min; yield: 91\%; Melting point: 208-210 ${ }^{\circ} \mathrm{C}^{17}$ FTIR $\left(\mathrm{KBr}, \mathrm{cm}^{-1}\right): v=1579.37(\mathrm{C}=\mathrm{C}), 1624.91(\mathrm{C}=\mathrm{N})$, $2188.78(\mathrm{CN}), 3353.51\left(\mathrm{NH}_{2}\right), 3448.78(\mathrm{NH}) ;{ }^{1} \mathrm{H}$ NMR (400 MHz, DMSO d6): $\delta=1.8\left(\mathrm{~s}, 3 \mathrm{H}, \mathrm{CH}_{3}\right)$, $2.28\left(\mathrm{~s}, 3 \mathrm{H}, \mathrm{Ar}_{-} \mathrm{CH}_{3}\right), 4.52$ (s, 1H, C-4 pyran), 6.85 (d, 2H, p-tolyl-H), 7.00 (d, 2H, p-tolyl-H), 7.18 (s, $2 \mathrm{H}, \mathrm{NH}_{2}$ ), 12.09 (s, $\left.1 \mathrm{H}, \mathrm{NH}\right) ;{ }^{13} \mathrm{C}$ NMR (75 MHz, DMSO-d6): $\delta=9.9,20.7,36.7,40.1,78.3,97.4$, 
120.7, 127.2, 128.6, 135.4, 140.9, 154.7, 160.6; Elemental analysis calculated for $\mathrm{C}_{15} \mathrm{H}_{14} \mathrm{~N}_{4} \mathrm{O}$ : C (67.65 \%), H (5.30 \%), N (21.04 \%). Found: C (67.15\%), H (5.17\%), N (21.00\%).

6-Amino-4-(4-methoxyphenyl)-3-methyl-1,4-dihydropyrano[2,3-c]pyrazole-5-carbonitrile (5c): Time: $35 \mathrm{~min}$; yield: 94\%; Melting point: $210-212{ }^{\circ} \mathrm{C} ;{ }^{15}{ }^{1} \mathrm{H}$ NMR $\left(400 \mathrm{MHz}, \mathrm{DMSO}-d_{6}\right) \delta 1.76\left(\mathrm{~s}, 3 \mathrm{H},-\mathrm{CH}_{3}\right)$, $3.71\left(\mathrm{~s}, 3 \mathrm{H},-\mathrm{OCH}_{3}\right), 4.51(\mathrm{~s}, 1 \mathrm{H}), 6.79\left(\mathrm{~s}, 2 \mathrm{H},-\mathrm{NH}_{2}\right), 6.84(\mathrm{~d}, 2 \mathrm{H}, J=8.0 \mathrm{~Hz}), 7.04(\mathrm{~d}, 2 \mathrm{H}, J=8.0$ $\mathrm{Hz}), 12.04(\mathrm{~s}, 1 \mathrm{H},-\mathrm{NH}) .{ }^{13} \mathrm{C} \mathrm{NMR}\left(50 \mathrm{MHz}, \mathrm{DMSO}-d_{6}+\mathrm{CDCl}_{3}\right) \delta .8 .8,34.7,53.8,57.7,96.5,112.5$, $119.7,127.4,134.7,134.8,153.8,157.0,159.5$. Mass (ES-MS) $m / z 283.2\left(\mathbf{M}^{+}\right)$.

6-Amino-4-(4-chlorophenyl)-3-methyl-1,4-dihydropyrano[2,3-c]pyrazole-5-carbonitrile (5d): Time: $35 \mathrm{~min}$; yield: 93\%; Melting point: $231-233^{\circ} \mathrm{C}^{15}$ Yellow solid, FTIR $\left(\mathrm{KBr}, \mathrm{cm}^{-1}\right): v=2228.22$, 3231.27, 3346.76, 3484.11; ${ }^{1} \mathrm{H}$ NMR (300 MHz, DMSO): $\delta 1.77$ (s, 3H, $\left.\mathrm{CH}_{3}\right), 4.67(\mathrm{~s}, 1 \mathrm{H},-\mathrm{CH}), 6.55$ (bs, $2 \mathrm{H},-\mathrm{NH}_{2}$ ), 7.35-7.37 (dd, 2H, Ar-H, $J=6.0 \mathrm{~Hz}$ ), 8.09-8.12 (dd, $2 \mathrm{H}, \mathrm{Ar}-\mathrm{H}, J=9.0 \mathrm{~Hz}$ ), 11.95 (s, $1 \mathrm{H},-\mathrm{NH}),{ }^{13} \mathrm{C}$ NMR $\left(75 \mathrm{MHz}, \mathrm{CDCl}_{3}\right): \delta 10.21,36.33,97.37,120.99,128.67,129.54,131.96,135.99$, 143.47, 155.19, 161.27; Elemental analysis calculated for $\mathrm{C}_{14} \mathrm{H}_{11} \mathrm{ClN} 4 \mathrm{O}: \mathrm{C}(55.39 \%) ; \mathrm{H},(4.62 \%)$; $\mathrm{N}$ (23.09\%). Found: C (55.36\%), H (4.65\%), N (23.06\%).

6-Amino-4-(2-chlorophenyl)-1,4-dihydro-3-methylpyrano[2,3-c]pyrazole-5-carbonitrile (5e): Time: 30 min; yield: 89\%; Melting point: $144-146^{\circ} \mathrm{C}^{27 \mathrm{~b}}$ FTIR $\left(\mathrm{KBr}, \mathrm{cm}^{-1}\right): v=1218,1407,1600,1651,2187$, 3313, 3413; ${ }^{1} \mathrm{H}$ NMR (DMSO-d $6, \delta$ ppm, $\left.J \mathrm{~Hz}\right): 1.8\left(\mathrm{~s}, 3 \mathrm{H}, \mathrm{CH}_{3}\right) ; 4.6(\mathrm{~s}, 1 \mathrm{H}, \mathrm{CH}) ; 7.08\left(\mathrm{~s}, 2 \mathrm{H}, \mathrm{NH}_{2}\right)$; $7.32(\mathrm{~d}, 1 \mathrm{H}, J=7.7, \mathrm{Ar}-\mathrm{H}) ; 7.47$ (t, $1 \mathrm{H}, J=7.5$, Ar-H); 7.54 (t, $1 \mathrm{H}, J=7.5, \mathrm{Ar}-\mathrm{H}) ; 7.83(\mathrm{~d}, 1 \mathrm{H}, J=$ 7.4, Ar-H); 12.25 (s, 1H, NH). ${ }^{13} \mathrm{C}$ NMR (DMSO-d $6, \delta$ ppm): 9.63, 31.57, 56.16, 96.50, 120.46, $123.37,124.91,127.99,132.25,134.07,135.93,148.24,155.09,161.33$.

6-Amino-4-(4-fluorophenyl)-3-methyl-1,4-dihydropyrano[2,3-c]pyrazole-5-carbonitrile (5f): Time: 30 min; yield: 93\%; Melting point: $244-246^{\circ} \mathrm{C}^{15}$ White powder; FTIR $\left(\mathrm{KBr}, \mathrm{cm}^{-1}\right): v=1395,1491$, 1591, 2198, 3090, 3226; ${ }^{1} \mathrm{H}$ NMR (300 MHz, DMSO-d 6 ): $\delta=1.79$ (s, 3H), 4.64 (s, 1H), $6.92(\mathrm{~s}, 2 \mathrm{H})$, 7.10-7.30 (m, 4H), 12.13 (s, 1H); ${ }^{13} \mathrm{C}$ NMR (75 MHz, DMSO-dd): $\delta=9.78,35.47,38.69,38.96,39.24$, 45.01, 49.29, 57.07, 97.53, 115.07, 115.36, 120.77, 129.33, 129.43, 135.67, 140.68, 140.72, 154.72, 159.37, 160.85; MS (ESI): $m / z=271.1(\mathrm{M}+\mathrm{H})^{+}$.

6-Amino-4-(3-bromophenyl)-1,4-dihydro-3-methylpyrano[2,3-c]pyrazole-5-carbonitrile (5g): Time: $40 \mathrm{~min}$; yield: $87 \%$; Melting point: $219-221^{\circ} \mathrm{C} .{ }^{15}$ FTIR $\left(\mathrm{KBr}, \mathrm{cm}^{-1}\right): v=1600,1610,1645,2195(\mathrm{C}$ $=\mathrm{N}), 3260(\mathrm{NH}), 3320,3400(\mathrm{C}=\mathrm{C}, \mathrm{C}=\mathrm{N})$; ${ }^{1} \mathrm{H}$ NMR: $2.3\left(\mathrm{~s}, 3 \mathrm{H}, \mathrm{CH}_{3}\right), 4.6(\mathrm{~s}, 1 \mathrm{H}$, pyran 4-H), $6.8(\mathrm{~s}$, $2 \mathrm{H}, \mathrm{NH}), 7.1(\mathrm{~m}, 4 \mathrm{H}$, phenyl protons) and $11.8(\mathrm{~s}, 1 \mathrm{H}, \mathrm{NH})$. Elemental analysis calculated for $\mathrm{C}_{14} \mathrm{H}_{11} \mathrm{BrN}_{4} \mathrm{O}$ C (50.77\%), H (3.35\%), N (16.92\%), Br. Found C (50.70\%), H (3.50\%), (N17.10\%).

6-Amino-4-(4-bromophenyl)-3-methyl-1,4-dihydropyrano[2,3-c]pyrazole-5-carbonitrile (5h): Time: $40 \mathrm{~min}$; yield: $90 \%$; Melting point: $176-178^{\circ} \mathrm{C}^{15}$ Yellow solid, FTIR $\left(\mathrm{KBr}, \mathrm{cm}^{-1}\right): v=2193.51$, 3271.99, 3431.74, 3486.37; ${ }^{1} \mathrm{H}$ NMR (200 MHz, DMSO): $\delta 1.82\left(\mathrm{~s}, 3 \mathrm{H},-\mathrm{CH}_{3}\right), 4.56(\mathrm{~s}, 1 \mathrm{H}, \mathrm{CH}), 6.57$ ( bs, 2H, $-\mathrm{NH}_{2}$ ), 7.10-7.14 (dd, 2H, Ar-H, J = 12.0 Hz), 7.42-7.46 (dd, 2H, Ar-H, J = 12.0 Hz), 11.96 $(\mathrm{s}, 1 \mathrm{H},-\mathrm{NH}),{ }^{13} \mathrm{C}$ NMR $(50 \mathrm{MHz}, \mathrm{DMSO}): \delta=9.75,36.93,57.07,96.75,119.85,120.52,120.52$, $129.39,131.08,136.52,143.33,154.71$ and 160.77; Elemental analysis calculated for $\mathrm{C}_{14} \mathrm{H}_{11} \mathrm{BrN}_{4} \mathrm{O}$ : C (50.76\%), H (3.38\%), N (16.95\%). Found C (50.77\%), H (3.35\%), N (16.92\%).

6-Amino-4-(4-hydroxyphenyl)-3-methyl-1,4-dihydropyrano[2,3-c]pyrazole-5-carbonitrile (5i): Time: 35 min; yield: 92\%; Melting point: $222-224^{\circ} \mathrm{C}^{, 17}$ Yellow solid; FTIR $\left(\mathrm{KBr}, \mathrm{cm}^{-1}\right): v=2223.29$, 3126.13, 3253.86, 3459.99; ${ }^{1} \mathrm{H}$ NMR (200 MHz, DMSO): $\delta 1.81\left(\mathrm{~s}, 3 \mathrm{H},-\mathrm{CH}_{3}\right), 4.44(\mathrm{~s}, 1 \mathrm{H},-\mathrm{CH})$, 6.48 ( bs, 2H, - $\mathrm{NH}_{2}$ ), 6.71 (dd, 2H, Ar-H), 6.94 (dd, 2H, Ar-H), 9.06 (bs, 1H, -OH), 11.88 (s, 1H, $\mathrm{NH}$ ), Elemental analysis calculated for $\mathrm{C}_{14} \mathrm{H}_{12} \mathrm{~N}_{4} \mathrm{O}_{2}$ : C (58.92\%), H (5.31\%), N (24.58\%). Found C, (58.94\%), H (5.30\%), N (24.55\%).

6-Amino-1,4-dihydro-3-methyl-4-(2-nitrophenyl)pyrano[2,3-c]pyrazole-5-carbonitrile (5j): Time: 35 min; yield: $93 \%$; Melting point: $220-222^{\circ} \mathrm{C}^{27 \mathrm{~b}}$ yellow solid. FTIR $\left(\mathrm{KBr}, \mathrm{cm}^{-1}\right): v=733,805,1349$, 
1400, 1491, 1525, 1652, 2195, 3117, 3224, 3473; ${ }^{1} \mathrm{H}$ NMR (300, $\left.\mathrm{CDCl}_{3}\right): \delta 1.82(\mathrm{~s}, 3 \mathrm{H}), 4.82(\mathrm{~s}, 1 \mathrm{H})$, $6.92(\mathrm{~s}, 2 \mathrm{H}), 7.59-7.81(\mathrm{~m}, \mathrm{H}), 8.01(\mathrm{~s}, 1 \mathrm{H}), 8.09(\mathrm{~d}, J=8.4 \mathrm{~Hz}, 1 \mathrm{H}), 12.13(\mathrm{~s}, 1 \mathrm{H})$.

6-Amino-1,4-dihydro-3-methyl-4-(3-nitrophenyl)pyrano[2,3-c]pyrazole-5-carbonitrile (5k): Time: 30 min; yield: 89\%; Melting point: $194-196^{\circ} \mathrm{C}^{15}{ }^{15}$ FTIR $\left(\mathrm{KBr}, \mathrm{cm}^{-1}\right): v=2193.51,3271.99,3431.74$, 3486.37; ${ }^{1} \mathrm{H}$ NMR (200 MHz, DMSO): $\delta 1.82$ (s, 3H, - $\mathrm{CH}_{3}$ ), 4.56 (s, $\left.1 \mathrm{H},-\mathrm{CH}\right), 6.57$ (bs, 2H, -NH2), 7.10-7.14 (dd, $2 \mathrm{H}, \mathrm{Ar}-\mathrm{H}, J=12.0 \mathrm{~Hz}), 7.42-7.46(\mathrm{dd}, 2 \mathrm{H}, \mathrm{Ar}-\mathrm{H}, J=12.0 \mathrm{~Hz}), 11.96(\mathrm{~s}, 1 \mathrm{H},-\mathrm{NH}),{ }^{13} \mathrm{C}$ NMR $(100 \mathrm{MHz}, \mathrm{DMSO}): \delta$ 9.75, 36.93, 57.07, 96.75, 119.85, 120.52, 120.52, 129.39, 131.08 , 136.52, 143.33, 154.71, 160.77; Elemental analysis calculated for $\mathrm{C}_{14} \mathrm{H}_{11} \mathrm{BrN}_{4} \mathrm{O}: \mathrm{C}(50.76 \%), \mathrm{H}$ (3.38\%), N (16.95\%). Found C (50.77\%), H (3.35\%), N (16.92\%).

6-Amino-3-methyl-4-(4-nitrophenyl)-1,4-dihydropyrano[2,3-c]pyrazole-5-carbonitrile (5l): Time: 40 min; yield: 90\%; Melting point: $249-251^{\circ} \mathrm{C} ;{ }^{15}$ Yellow solid; FTIR $\left(\mathrm{KBr}, \mathrm{cm}^{-1}\right): v=1643.37,2189.52$, 3177.13, 3307.11, 3386.6; ${ }^{1} \mathrm{H}$ NMR (300 MHz, DMSO): $\delta 1.76$ (s,3H, $\left.-\mathrm{CH}_{3}\right), 4.65$ (s, $\left.1 \mathrm{H},-\mathrm{CH}\right), 6.35$ (bs, 2H, $-\mathrm{NH}_{2}$ ), 7.33-7.36 (dd, 2H, Ar-H, $J=9.0 \mathrm{~Hz}$ ), 8.08-8.11 (dd, 2H, Ar-H $J=9.0 \mathrm{~Hz}$ ), 11.90 (s, $1 \mathrm{H},-\mathrm{NH}),{ }^{13} \mathrm{C}$ NMR $\left(75 \mathrm{MHz}, \mathrm{CDCl}_{3}\right): \delta 10.16,36.73,58.01,96.39,120.49,123.88,128.73,136.43$, $146.88,151.22,155.18,161.17$; Elemental analysis calculated for $\mathrm{C}_{14} \mathrm{H}_{11} \mathrm{~N}_{5} \mathrm{O}_{3}: \mathrm{C}(53.48 \%), \mathrm{H}$ (4.52\%), N (26.75\%). Found C (53.50\%), H (4.49\%), N (26.74\%).

6-Amino-4-(3,4-dimethoxyphenyl)-3-methyl-1,4-dihydropyrano[2,3-c]pyrazole-5-carbonitrile (5m): Time: $40 \mathrm{~min}$; yield: $84 \%$; Melting point: $192-194^{\circ} \mathrm{C} ;{ }^{; 7 b}$ Yellow solid, FTIR $\left(\mathrm{KBr}, \mathrm{cm}^{-1}\right): v=2186.78$, 3176.29, 3350.11, 3413.28; ${ }^{1} \mathrm{H}$ NMR (300 MHz, DMSO): $\delta 1.76\left(\mathrm{~s}, 3 \mathrm{H},-\mathrm{CH}_{3}\right), 3.71\left(\mathrm{~s}, 6 \mathrm{H},\left(\mathrm{OCH}_{3}\right)_{2}\right)$, $4.45(\mathrm{~s}, 1 \mathrm{H},-\mathrm{CH}), 6.37$ ( bs, 2H, $\left.-\mathrm{NH}_{2}\right), 6.75-6.78(\mathrm{dd}, 2 \mathrm{H}, \mathrm{Ar}-\mathrm{H}, J=9.0 \mathrm{~Hz}), 7.02-7.04$ (dd, $2 \mathrm{H}, \mathrm{Ar}-$ $\mathrm{H}), 11.84(\mathrm{~s}, 1 \mathrm{H},-\mathrm{NH})$; Elemental analysis calculated for $\mathrm{C}_{16} \mathrm{H}_{16} \mathrm{~N}_{4} \mathrm{O}_{3}: \mathrm{C}(58.37 \%), \mathrm{H}(5.84 \%), \mathrm{N}$ (21.25\%). Found C (58.35\%), H (5.81\%), N (21.26\%).

6-Amino-4-(4-hydroxy-3-methoxyphenyl)-3-methyl-1,4-dihydropyrano[2,3-c]pyrazole-5-carbonitrile $(5 \boldsymbol{n})$ : Time: $45 \mathrm{~min}$; yield: $88 \%$; Melting point: $236-238^{\circ} \mathrm{C} ;{ }^{15}$ Yellow solid, FTIR $\left(\mathrm{KBr}, \mathrm{cm}^{-1}\right): v=$ 2195.64, 3275.81, 3413.72, 3490.79; ${ }^{1} \mathrm{H}$ NMR (200 MHz, DMSO): $\delta 1.85$ (s, 3H, - $\left.\mathrm{CH}_{3}\right), 3.79$ (s, 3H,$\left.\mathrm{OCH}_{3}\right), 4.47$ (s, 1H, -CH), 6.18 (bs, 2H, - $\left.\mathrm{NH}_{2}\right), 6.66$ (m, 2H, Ar-H), 7.86 (s, 1H, Ar-H), 8.46 (bs, 1H,$\mathrm{OH}), 11.82(\mathrm{~s}, 1 \mathrm{H},-\mathrm{NH}),{ }^{13} \mathrm{C}$ NMR $(50 \mathrm{MHz}, \mathrm{DMSO}): \delta=9.78,26.15,53.37,86.18,97.46,114.92$, 119.74, 134.91, 140.13, 145.01, 154.79, 180.42, 186.13, 196.73, 211.13; Elemental analysis calculated for $\mathrm{C}_{15} \mathrm{H}_{14} \mathrm{~N}_{4} \mathrm{O}_{3}$ : C (57.16\%), $\mathrm{H}(5.42 \%), \mathrm{N}(22.19 \%)$. Found $\mathrm{C}(57.13 \%), \mathrm{H}(5.43 \%), \mathrm{N}(22.21 \%)$.

6-Amino-4-(4-(dimethylamino)phenyl)-3-methyl-1,4-dihydropyrano[2,3-c]pyrazole-5-carbonitrile (5o): Time: $40 \mathrm{~min}$; yield: $92 \%$; Melting point: $165-167^{\circ} \mathrm{C}$; ${ }^{27 \mathrm{~b}}$ Yellow solid, FTIR $\left(\mathrm{KBr}, \mathrm{cm}^{-1}\right)$ : v $=3441.70,3142.41,2173.69 ;{ }^{1} \mathrm{H}$ NMR $(300 \mathrm{MHz}, \mathrm{DMSO}): \delta 1.78\left(\mathrm{~s}, 3 \mathrm{H},-\mathrm{CH}_{3}\right), 2.86(\mathrm{~s}, 6 \mathrm{H},-$ $\left(\mathrm{N}\left(\mathrm{CH}_{3}\right)_{2}\right), 4.41(\mathrm{~s}, 1 \mathrm{H},-\mathrm{CH}), 6.01-6.62\left(\mathrm{~m}, 4 \mathrm{H}, \mathrm{Ar}-\mathrm{H},-\mathrm{NH}_{2}\right), 6.94-6.97(\mathrm{dd}, 2 \mathrm{H}, \mathrm{Ar}-\mathrm{H}, J=9.0 \mathrm{~Hz})$, 8.08-8.11 (dd, 2H, Ar-H, J=9.0 Hz), $11.91(\mathrm{~s}, 1 \mathrm{H}, \mathrm{NH}),{ }^{13} \mathrm{C}$ NMR $\left(75 \mathrm{MHz}, \mathrm{CDCl}_{3}\right)(\mathrm{Fig} .4 .13): \delta$ 10.22, 35.97, 58.83, 98.43, 112.61, 121.34, 128.37, 132.30, 135.91, 149.56, 155.26, 160.95; Elemental analysis calculated for $\mathrm{C}_{16} \mathrm{H}_{17} \mathrm{~N}_{5} \mathrm{O}: \mathrm{C}(61.55 \%), \mathrm{H}(6.43 \%), \mathrm{N}(26.88 \%)$. Found $\mathrm{C}(61.52 \%), \mathrm{H}$ $(6.45 \%), \mathrm{N},(26.90 \%)$.

6-Amino-4-(furan-2-yl)-1,4-dihydro-3-methylpyrano[2,3-c]pyrazole-5-carbonitrile (5p): Time: 30 min; yield: $90 \%$; Melting point: $235-238^{\circ} \mathrm{C} .^{27 \mathrm{~b}}$

6-Amino-1,4-dihydro-3-methyl-4-(thiophen-2-yl)pyrano[2,3-c]pyrazole-5-carbonitrile (5q): Time: 40 min; yield: $90 \%$; Melting point: $234-237^{\circ} \mathrm{C} .^{27 b}$

\subsection{Biological Assay}

\subsubsection{Antibacterial Activity}

For bacterial strain $S$. aureus, it can be seen that, the compounds $5 \mathbf{d}, \mathbf{5 e}, \mathbf{5 f}$ and $\mathbf{5 j}$ showed excellent inhibitory activity with MIC value $4 \mu \mathrm{g} / \mathrm{mL}$, which is equivalent to ampicilin (MIC 4 
$\mu \mathrm{g} / \mathrm{mL}$ ). For bacterial strain $M$. luteus, compounds $\mathbf{5 c}, \mathbf{5 f}, \mathbf{5 h}, \mathbf{5 i}, \mathbf{5 k}$ and $\mathbf{5 l}$ exhibited four-fold antibacterial activity with MIC value $4 \mu \mathrm{g} / \mathrm{mL}$ and compounds $\mathbf{5 d}, \mathbf{5 j}, \mathbf{5 m}$ and $\mathbf{5 p}$ with MIC value 8 $\mu \mathrm{g} / \mathrm{mL}$ exhibited two-fold more activity as compared to the clinical drug ampicilin (MIC $16 \mu \mathrm{g} / \mathrm{mL}$ ). For bacterial strain E. coli and P. fluorescens, all the synthesized compounds exhibited moderate antibacterial activity compared to the standard drug (Table 3 ).

\subsubsection{Antifungal Activity}

Compounds $\mathbf{5 b}, \mathbf{5 c}, \mathbf{5 h}$ and $\mathbf{5 k}$ with MIC value $4 \mu \mathrm{g} / \mathrm{mL}$ exhibited four-fold more activity compared with the standard drug miconazole and compounds $\mathbf{5 d}, \mathbf{5 f}, \mathbf{5 l}, \mathbf{5 m}, \mathbf{5 o}$ and $\mathbf{5 p}$ with MIC value $8 \mu \mathrm{g} / \mathrm{mL}$ exhibited two-fold more activity compared to the miconazole against the fungicidal strain $C$. albicans. Compounds $5 \mathbf{5 a}, \mathbf{5 e}, \mathbf{5 g}, \mathbf{5 i}, \mathbf{5 j}, \mathbf{5 n}$ and $\mathbf{5 q}$ with MIC value $16 \mu \mathrm{g} / \mathrm{mL}$ exhibited equivalent activity compared with the standard drug miconazole. Compounds $\mathbf{5 c}$ and $\mathbf{5 f}$ with MIC value $4 \mu \mathrm{g} / \mathrm{mL}$ exhibited four-fold more activity compared with the standard drug miconazole and compounds $\mathbf{5 b}, \mathbf{5 h}, \mathbf{5 j}, \mathbf{5 k}, \mathbf{5 l}$ and $\mathbf{5 p}$ with MIC value $8 \mu \mathrm{g} / \mathrm{mL}$ exhibited two-fold more activity compared to the miconazole for the fungicidal strain $F$. oxysporum. Compounds $5 \mathbf{a}, \mathbf{5 d}, \mathbf{5 e}, \mathbf{5 g}, \mathbf{5 m}$, 5n, 5o and 5q with MIC value $16 \mu \mathrm{g} / \mathrm{mL}$ exhibited equivalent activity compared with the standard drug miconazole. Compounds 5k with MIC value $4 \mu \mathrm{g} / \mathrm{mL}$ exhibited four-fold more activity compared with the standard drug miconazole and compounds $\mathbf{5 b}, \mathbf{5 c}, \mathbf{5 d}, \mathbf{5 f}, \mathbf{5 h}, \mathbf{5 j}, \mathbf{5 l}$ and $\mathbf{5 m}$ with MIC value $8 \mu \mathrm{g} / \mathrm{mL}$ exhibited two-fold more activity compared to the miconazole against the fungicidal strain A. flavus. Compounds 5a, 5e, 5i, 5n, 5o, 5p and 5q with MIC value $16 \mu \mathrm{g} / \mathrm{mL}$ exhibited equivalent activity compared with the standard drug miconazole (Table 3 ).

Table 3. In vitro antimicrobial and antioxidant activities of compounds $\mathbf{5 a - q}(\mu \mathrm{g} / \mathrm{mL})$

\begin{tabular}{|c|c|c|c|c|c|c|c|c|}
\hline \multirow[t]{2}{*}{ Compounds } & \multicolumn{2}{|c|}{$\begin{array}{c}\text { Gram positive } \\
\text { bacteria } \text { IC }_{50}\end{array}$} & \multicolumn{2}{|c|}{$\begin{array}{c}\text { Gram negative } \\
\text { bacteria } \text { IC }_{50}\end{array}$} & \multicolumn{3}{|c|}{ Antifungal activity $\mathrm{IC}_{50}$} & \multirow{2}{*}{$\begin{array}{c}\text { DPPH } \\
\text { IC }_{50}\end{array}$} \\
\hline & $\mathbf{S A}$ & ML & EC & PF & CA & FO & $\mathbf{A F}$ & \\
\hline $5 a$ & 32 & 32 & 32 & 16 & 16 & 16 & 16 & 22.1 \\
\hline $5 b$ & 16 & 16 & 16 & 16 & 4 & 8 & 8 & 27.3 \\
\hline $5 c$ & 16 & 4 & 8 & 8 & 4 & 4 & 8 & 23.1 \\
\hline $5 d$ & 4 & 8 & 8 & 8 & 8 & 16 & 8 & 20.1 \\
\hline $5 e$ & 4 & 32 & 8 & 8 & 16 & 16 & 16 & 18.3 \\
\hline $5 f$ & 4 & 4 & 8 & 8 & 8 & 4 & 8 & 14.1 \\
\hline $5 g$ & 16 & 16 & 8 & 8 & 16 & 16 & 32 & 18.1 \\
\hline $5 \mathrm{~h}$ & 16 & 4 & 16 & 16 & 4 & 8 & 8 & 15.3 \\
\hline $5 \mathbf{i}$ & 8 & 4 & 16 & 16 & 16 & 32 & 16 & 10.3 \\
\hline $5 \mathbf{j}$ & 4 & 8 & 16 & 16 & 16 & 8 & 8 & 21.3 \\
\hline $5 \mathbf{k}$ & 16 & 4 & 8 & 4 & 4 & 8 & 4 & 19.2 \\
\hline 51 & 8 & 4 & 8 & 16 & 8 & 8 & 8 & 21.3 \\
\hline $5 \mathrm{~m}$ & 8 & 8 & 8 & 16 & 8 & 16 & 8 & 24.1 \\
\hline $5 n$ & 16 & 16 & 16 & 8 & 16 & 16 & 16 & 10.2 \\
\hline 50 & 32 & 16 & 8 & 8 & 8 & 16 & 16 & 24.6 \\
\hline $5 p$ & 16 & 8 & 8 & 16 & 8 & 8 & 16 & 28.8 \\
\hline $5 q$ & 16 & 16 & 16 & 8 & 16 & 16 & 16 & 29.3 \\
\hline Ampicilin & 4 & 16 & 4 & 2 & NA & NA & NA & NA \\
\hline Kanamycin & 2 & 2 & 2 & 2 & - & NA & NA & NA \\
\hline Miconazole & NA & NA & NA & NA & 16 & 16 & 16 & NA \\
\hline Fluconazole & NA & NA & NA & NA & 2 & 2 & 4 & NA \\
\hline BHT & NA & NA & NA & NA & $\mathrm{NA}$ & NA & NA & 16.5 \\
\hline
\end{tabular}

SA: Staphylococcus aureus; ML: Micrococcus luteus; EC: Escherichia coli; PF: Pseudomonas fluorescens; CA: Candida albicans; FO: Fusarium oxysporum; AF: Aspergillus flavus; BHT: Butylated hydroxy toluene; NA: Not applicable

\subsubsection{Antioxidant Activity}

All the synthesized compounds 5a-q shows good to moderate antioxidant activity as compared to the standard drug BHT (Table 3). The compounds 5i and 5n with -OH substituent on phenyl ring have shown excellent activity as compared to standard drug. Again, the compound $\mathbf{5 f}(14.1 \mu \mathrm{g} / \mathrm{mL})$ with fluoro- group and $\mathbf{5 h}(15.3 \mu \mathrm{g} / \mathrm{mL})$ with bromo- group showed excellent antioxidant activity as 
compared to the BHT. Remaining compounds exhibit well to moderate antioxidant activity as compared to standard drug BHT (Table 3).

\subsection{Computational Chemistry}

\subsubsection{In silico ADME Prediction}

It is observed that, the compounds exhibited a good \% ABS (\% absorption) ranging from 62.92 to $78.73 \%$ (Table 4). Furthermore, none of the compounds violated Lipinski's rule of five (miLog $P \leq 5$ ). A molecule likely to be developed as an orally active drug candidate should show no more than one violation of the following four criteria: miLog $P$ (octanol-water partition coefficient) $\leq$ 5 , molecular weight $\leq 500$, number of hydrogen bond acceptors $\leq 10$ and number of hydrogen bond donors $\leq 5 .{ }^{39}$ The larger the value of the drug likeness model score, the higher is also probability that the particular molecule will be active. All the tested compounds followed the criteria for orally active drug and therefore, these compounds may have a good potential for eventual development as oral agents.

$\underline{\text { Table 4. Pharmacokinetic parameters important for good oral bioavailability }}$

\begin{tabular}{|c|c|c|c|c|c|c|c|c|c|c|}
\hline Comp. & $\underset{\%}{\mathrm{ABS}}$ & $\begin{array}{c}\text { TPSA } \\
\left(\mathbf{A}^{2}\right)\end{array}$ & $\begin{array}{c}\text { n- } \\
\text { ROTB }\end{array}$ & MV & MW & $\operatorname{miLog} P$ & n-ON & n-OHNH & $\begin{array}{l}\text { Lipinski } \\
\text { violation }\end{array}$ & $\begin{array}{c}\text { Drug- } \\
\text { likeness } \\
\text { model } \\
\text { score }\end{array}$ \\
\hline Rule & - & - & - & - & $<500$ & $\leq 5$ & $<10$ & $<5$ & $\leq 1$ & - \\
\hline $5 a$ & 78.73 & 87.73 & 1 & 223.38 & 252.28 & 1.44 & 5 & 3 & 0 & -0.16 \\
\hline $5 b$ & 78.73 & 87.73 & 1 & 239.94 & 266.30 & 1.89 & 5 & 3 & 0 & -0.26 \\
\hline $5 c$ & 75.54 & 96.97 & 2 & 248.92 & 282.30 & 1.50 & 6 & 3 & 0 & 0.05 \\
\hline $5 d$ & 78.73 & 87.73 & 1 & 236.91 & 286.72 & 2.12 & 5 & 3 & 0 & 0.29 \\
\hline $5 e$ & 78.73 & 87.73 & 1 & 236.91 & 286.72 & 2.07 & 5 & 3 & 0 & 0.19 \\
\hline $5 f$ & 78.73 & 87.73 & 1 & 228.31 & 270.27 & 1.61 & 5 & 3 & 0 & 0.13 \\
\hline $5 \mathrm{~g}$ & 78.73 & 87.73 & 1 & 241.26 & 331.17 & 2.23 & 5 & 3 & 0 & -0.23 \\
\hline $5 \mathrm{~h}$ & 78.73 & 87.73 & 1 & 241.26 & 331.17 & 2.25 & 5 & 3 & 0 & -0.06 \\
\hline $5 i$ & 71.75 & 107.96 & 1 & 231.40 & 268.28 & 0.96 & 6 & 4 & 0 & 0.24 \\
\hline $5 j$ & 62.92 & 133.56 & 2 & 246.71 & 297.27 & 1.35 & 8 & 3 & 0 & -0.22 \\
\hline $5 \mathbf{k}$ & 62.92 & 133.56 & 2 & 246.71 & 297.27 & 1.38 & 8 & 3 & 0 & -0.11 \\
\hline 51 & 62.92 & 133.56 & 2 & 246.71 & 297.27 & 1.40 & 8 & 3 & 0 & -0.18 \\
\hline $5 \mathrm{~m}$ & 72.36 & 106.20 & 3 & 274.47 & 312.33 & 1.09 & 7 & 3 & 0 & 0.43 \\
\hline $5 n$ & 68.56 & 117.19 & 2 & 256.94 & 298.30 & 0.78 & 7 & 4 & 0 & 0.51 \\
\hline 50 & 77.62 & 90.97 & 2 & 269.28 & 295.35 & 1.55 & 6 & 3 & 0 & -0.25 \\
\hline $5 p$ & 74.20 & 100.87 & 1 & 204.95 & 242.24 & 0.70 & 6 & 3 & 0 & -0.23 \\
\hline $5 q$ & 78.73 & 87.73 & 1 & 214.09 & 258.31 & 1.34 & 5 & 3 & 0 & -0.11 \\
\hline
\end{tabular}

\section{Conclusion}

A facile, eco-friendly and green protocol developed for one-pot multicomponent cyclocondensation of aldehydes, malononitrile, hydrazine hydrate and ethyl acetoacetate is established. Application of gluconic acid aqueous solution (GAAS) as a reaction medium for the synthesis of pyrano $[2,3-c]$ pyrazoles has been exploited first time. The reaction conditions are mild accepting several functional groups present in the molecules and all reactions proceed under essentially neutral conditions, thus reducing the possibility of many unwanted side reactions. In addition, present method offers marked improvements with regard to product yield, reaction time, and greenness of procedure, avoiding hazardous organic solvents/toxic catalysts and provides a better, clean and practical alternative route of synthesis to the existing protocols. The synthesized Dihydropyrano [2,3-c] pyrazoles were evaluated for antimicrobial and antioxidant activity and also analyzed for ADME properties. 


\section{Acknowledgements}

The authors are very thankful to Department of Chemistry, Dr. Rafiq Zakaria, college for women, Aurangabad for providing facility and support to furnish our work.

\section{Supporting Information}

Supporting information accompanies this paper on http://www.acgpubs.org/journal/organic$\underline{\text { communications }}$

\section{ORCID}

Furqan Diwan: 0000-0001-7101-8337

Mubarak H. Shaikh: 0000-0002-1190-2371

Samreen Fatema: 0000-0002-7721-8089

Mazahar Farooqui: 0000-0003-2236-6639

\section{References}

[1] Canete-Rodriguez, A. M.; Santos-Duena, I. M.; Jimenez-Hornero, J. E.; Ehrenreich, A.; Liebl, W.; Garcia, I. G. Gluconic acid: Properties, production methods and applications-An excellent opportunity for agro-industrial by-products and waste bio-valorization. Proc. Biochem. 2016, 51, 1891-1903.

[2] (a) Yang, J.; Zhou, B.; Li, M.; Gu, Y. Gluconic acid aqueous solution: a task-specific bio-based solvent for ring-opening reactions of dihydropyrans. Tetrahedron 2013, 69, 1057-1064; (b) Zhou, B.; Yang, J.; Li, M.; Gu, Y. Gluconic acid aqueous solution as a sustainable and recyclable promoting medium for organic reactions. Green Chem. 2011, 13, 2204-2211.

[3] Mahmoud, N. F. H.; El-bordany, E. A.; Elsayed, G. A. Synthesis and pharmacological activities of pyrano[2,3- $d]$ pyrimidine and pyrano[2,3- $d]$ pyrimidine-5-one derivatives as new fused heterocyclic systems. J. Chem. 2017, 1-7.

[4] Ambethkar, S.; Padmini, V.; Bhuvanesh, N. A green and efficient protocol for the synthesis of dihydropyrano[2,3-c]pyrazole derivatives via a one-pot, four component reaction by grinding method. $J$. Adv. Res. 2015, 6, 975-985.

[5] Ismail, Z. H.; Aly, G. M.; El-Degwi, M.S.; Heiba, H. I.; Ghorab, M. M. Synthesis and insecticidal activity of some new pyranopyrazoles, pyrazolopyranopyrimidines, and pyrazolopyranopyridines. Egypt J. Biotechnol. 2003, 13, 73-82

[6] (a) Abdelrazek, F. M.; Metz, P.; Metwally, N. H.; El-Mahrouky, S. F. Synthesis and molluscicidal activity of new cinnoline and pyrano [2,3-c]pyrazole derivatives. Arch. Pharm. 2006, 339, 456-460;

(b) Abdelrazek, F. M.; Metz, P.; Kataeva, O.; Jaeger, A.; El-Mahrouky, S. F. Synthesis and molluscicidal activity of new chromene and pyrano[2,3-c]pyrazole derivatives. Arch Pharm. 2007, 340, 543-548.

[7] Kuo, S. C.; Huang, L. J.; Nakamura, H. Synthesis and analgesic and antiinflammatory activities of 3,4dimethylpyrano[2,3-c]pyrazol-6-one derivatives. J. Med. Chem. 1984, 27, 539-544.

[8] Zaki, M. E. A.; Soliman, H. A.; Hiekal, O. A.; Rashad, A. E. Z. Pyrazolopyranopyrimidines as a class of anti-inflammatory agents. Z. Naturforsch C. 2006, 61, 1-5.

[9] Ahluwalia, V. K.; Dahiya, A.; Garg, V. Reaction of 5-amino-4-formyl-3-methyl(or phenyl)-1-phenyl-1Hpyrazoles with active methylene compounds: Synthesis of fused heterocyclic rings. Ind. J. Chem. 1997, $36,88-90$

[10] (a) Nadia, M. R.; Nahed, Y. K.; Fahmyb, A.; El-Sayeda, A. A. F. Facile synthesis of fused nitrogen containing heterocycles as anticancer agents. Der Pharm. Chemica. 2010, 2, 400-417.; (b) Wang, J. L.; Liu, D.; Zheng, Z. J.; Shan, S.; Han, X.; Srinivasula, S. M.; Croce, C. M.; Alnemri, E. S.; Huang, Z. Structure-based discovery of an organic compound that binds Bcl-2 protein and induces apoptosis of tumor cells. Proc. Natl. Acad. Sci. USA. 2000, 97, 7124-7129.

[11] Foloppe, N.; Fisher, L. M.; Howes, R.; Potter, A.; Robertson, A. G. S.; Surgenor, A. E. Identification of chemically diverse Chk1 inhibitors by receptor-based virtual screening. Bioorg. Med. Chem. 2006, 14, 4792-4802. 
[12] Stachulski, A. V.; Berry, N. G.; Low, A. C. L.; Moores, S. L.; Row, E.; Warhurst, D. C.; Adagu, I. S.; Rossignol, J. F. Identification of isoflavone derivatives as effective anticryptosporidial agents in vitro and in vivo. J. Med. Chem. 2006, 49, 1450-1454.

[13] Khodja, I. M.; Fisli, A.; Lebhour, O.; Boulcina, R.; Boumoud, B.; Debache, A. Four-Component synthesis of pyrano[2,3-c]pyrazoles catalyzed by triphenylphosphine in aqueous medium. Lett. Org. Chem. 2016, 13, 85-91.

[14] Li, W.; Ruzi.; Ablajan, R. K.; Ghalipt, Z. One-pot synthesis of highly functionalized pyrano[2,3c]pyrazole-4,4'-diacetate and 6-oxo-pyrano[2,3-c]pyrazole derivatives catalyzed by urea. Tetrahedron 2017, 73, 164-171.

[15] Zakeri, M.; Nasef, M. M.; Kargaran, ; Ahmad, T. A.; Lotf, E. A.; Asadi, J. Synthesis of pyrano[2,3c]pyrazoles by ionic liquids under green and eco-safe conditions. Res. Chem. Intermed. 2017, 43, 717728.

[16] Vasuki, G.; Kumaravel, K. Rapid four-component reactions in water: synthesis of pyranopyrazoles. Tetrahedron Lett. 2008, 49, 5636-5638.

[17] Wu, M.; Feng, Q.; Wan, D.; Ma, J. CTACl as catalyst for four-component, one-pot synthesis of pyranopyrazole derivatives in aqueous medium. Synth. Commun. 2013, 43, 1721-1726.

[18] Heravi, M. M.; Ghods A.; Derikvand, F.; Bakhtiari, K.; Bammoharram, F. F. H14[NaP5W30O110] catalyzed one-pot three-component synthesis of dihydropyrano[2,3-c]pyrazole and pyrano[2,3d]pyrimidine derivatives. J. Iran. Chem. Soc. 2010, 7, 615-620.

[19] Parmar, N. J.; Barad, H. A.; Pansuriya B. R.; Talpada, N. P. A highly efficient, rapid one-pot synthesis of some new heteroaryl pyrano[2,3-c]pyrazoles in ionic liquid under microwave-irradiation. RSC Adv. 2013, 3, 8064-8070.

[20] Peng, Y.; Song, G.; Dou, R. Surface cleaning under combined microwave and ultrasound irradiation: flash synthesis of 4H-pyrano[2,3-c]pyrazoles in aqueous media. Green Chem. 2006, 8, 573-575.

[21] Lehmann, F.; HolmMand Laufer, S. Three-Component combinatorial synthesis of novel dihydropyrano[2,3-c]pyrazoles. J. Comb. Chem. 2008, 10, 364-367.

[22] Mecadon, H.; Rohman, M. R.; Kharbangar, I.; Laloo, B. M.; Kharkongor, I.; Rajbangshi M.; Myrboh, B. Tetrahedron Lett. 2011, 52, 3228-3231.

[23] Mecadon, H.; Rohman, M. R.; Rajbangshi M.; Myrboh, B. $\gamma$-Alumina as a recyclable catalyst for the four-component synthesis of 6-amino-4-alkyl/aryl-3-methyl-2,4-dihydropyrano[2,3-c]pyrazole-5carbonitriles in aqueous medium. Tetrahedron Lett. 2011, 52, 2523-2525.

[24] Kanagaraj, K.; Pitchumani, K. Solvent-Free multicomponent synthesis of pyranopyrazoles: per-6-aminobcyclodextrin as a remarkable catalyst and host. Tetrahedron Lett. 2010, 51, 3312-3316.

[25] Kiyania, H.; Samimib, H. A.; Ghorbania, F.; Esmaielia, S. One-pot, four-component synthesis of pyrano[2,3-c]pyrazoles catalyzed by sodium benzoate in aqueous medium. Curr. Chem. Lett. 2013, 2, 197-206.

[26] Bihani, M.; Bora, P. P.; Bez, G.; Askari, H. Amberlyst A21 catalyzed chromatography-free method for multicomponent synthesis of dihydropyrano[2,3-c]pyrazoles in ethanol. ACS Sustain. Chem. Eng. 2013, 1, 440-447.

[27] (a ) Madhusudana Reddy, M. B.; Jayashan Kara, V. P.; Pasha, M. A. Glycine-catalyzed efficient synthesis of pyranopyrazoles via one-pot multicomponent reaction. Synth. Commun. 2010, 40, 2930-2934.; (b) Nikam, M. D.; Mahajan, P. S.; Chate, A. V.; Dabhade, S. K.; Gill, C. H. An efficient and green protocol for the synthesis of dihydropyrano [2,3-c] pyrazoles in aqueous medium using thiamine hydrochloride as a catalyst. J. Chil. Chem. Soc. 2015, 60, 2847-2850.

[28] Siddekha, A.; Nizam, A.; Pasha, M. A. An efficient and simple approach for the synthesis of pyranopyrazoles using imidazole (catalytic) in aqueous medium, and the vibrational spectroscopic studies on 6-amino-4-(4-methoxyphenyl)-5-cyano-3-methyl-1-phenyl-1,4-dihydropyrano[2,3-c]pyrazole using density functional theory. Spectrochim Act. Part A, 2011, 81, 431-440.

[29] Madhusudana Reddy, M.B.; Pasha, M.A. One-pot, multicomponent synthesis of 4H-pyrano[2,3c]pyrazoles in water at $25^{\circ} \mathrm{C}$. Ind. J. Chem. 2012, 51, 537-541.

[30] (a) Diwan, F.; Farooqui, M. $\gamma$-Valerolactone as a promising bio-compatible media for one-pot synthesis of spiro[indoline-3,4'-pyrano[3,2-c]chromene derivatives. J. Het. Chem. 2018, 55, 2817-2822; (b) Diwan, F.; Shaikh, M.; Farooqui, M. Lemon juice catalyzed efficient one-pot synthesis, antioxidant and antimicrobial evaluation of bispyrazolyl methanes. Chem. \& Biol. Interface 2018, 8, 255-268; (c) Diwan, F.; Shaikh, M. H.; Shaikh, M.; Farooqui, M. $\gamma$-Valerolactone: Promising bio-compatible media for the synthesis of 2-arylbenzothiazole derivatives. Org. Commun. 2019, 12, 1-13. 
[31] (a) NCCLS (National Committee for Clinical Laboratory Standards), Performance Standards for Antimicrobial Susceptibility Testing: Twelfth Informational Supplement, 2002, 1-56238-454-6 M100S12(M7). (b) Lai, P; Rao, H; Gao, Y. Chemical composition, cytotoxic, antimicrobial and antioxidant activities of essential oil from Anthriscus caucalis M. Bieb grown in China. Rec.Nat.Prod. 2018, 12(4), 290-294.

[32] (a) Greenwood, D.; Slack, R.C.B.; Peutherer, J.F. Medical Microbiology. 14th ed., ELBS with Churchill Livingstone, London, 1992. (b) Suryavanshi, H.R.; Rathore, M.M. Synthesis and biological activities of piperazine derivatives as antimicrobial and antifungal agents. Org.Commun. 2017,10(3), 228-238.

[33] Han, H.; Yllmaz, H; Gulcin, I. Antioxidant activity of flaxseed (Linum usitatissimum L.) shell and analysis of its polyphenol contents by LC-MS/MS. Rec.Nat.Prod. 2018, 12(4), 397-402.

[34] Lipinski, C. A.; Lombardo, L.; Dominy, B. W.; Feeney, P. J. Experimental and computational approaches to estimate solubility and permeability in drug discovery and development settings Adv. Drug Deliv. Rev. 2001, 46, 3-26.

[35] Molinspiration Chemoinformatics Brastislava, Slovak Republic, Available from: http://www.molinspiration.com/cgi-bin/properties 2014.

[36] Zhao, Y. H.; Abraham, M. H.; Le, J.; Hersey, A.; Luscombe, N. C.; Beck, G.; Sherborne, B.; Cooper, I. Rate-limited steps of human oral absorption and QSAR studies. Pharm. Res. 2002, 19, 1446-1457.

[37] Drug-likeness and molecular property prediction, available from: http://www.molsoft.com/mprop/

[38] Al-Matar, H. M.; Khalil, K. D.; Meier, H.; Kolshorn, H.; Elnagdi, M. H. Chitosan as heterogeneous catalyst in Michael additions: the reaction of cinnamonitriles with active methylene moieties and phenols. Arkivoc, 2008, xvi, 288-301.

[39] Ertl, P.; Rohde, B.; Selzer, P. Fast calculation of molecular polar surface area as a sum of fragment-based contributions and Its application to the prediction of drug transport properties. J. Med. Chem. 2000, 43, 3714-3717.

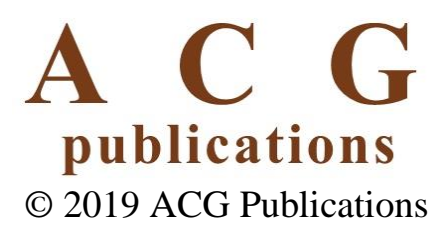

